\title{
Publish Or Perish: \\ Evaluating And Promoting Scholarly Output
}

Low Hui Min, Ph.D., Universiti Sains Malaysia, Malaysia

Amelia Abdullah, Ph.D., Universiti Sains Malaysia, Malaysia

Abdul Rashid Mohamed, Ph.D., Universiti Sains Malaysia, Malaysia

\begin{abstract}
Scholarly output, particularly in the form of journal publication is a key indicator in various levels of university performance. It contributes to university ranking, faculty ranking and academicians' scholarship credentials. Therefore, scholarly output has become a standard measure used to determine intake, promotion and tenure renewal of academicians at the faculty and university levels. In this paper, the Dean's office of an education faculty in a Malaysia university collected and analyzed the faculty members' journal publication outputs in 2011 as a way to determine the directions to move forward. From an integrated quantitative and qualitative investigation, research supervision and group publication were identified as the major catalysts for research publications. The findings informed the importance of project development and training in these two areas.
\end{abstract}

Keywords: Publish or Perish; Evaluating Scholarly Output

\section{INTRODUCTION}

$\varnothing$

ournal publication is now emerging as the most prominent scholarly output to showcase scholarship credentials amongst the academicians. This publication output has almost replaced the traditional book publication (Lyytinen, Baskerville, Livari, \& Te'eni, 2007) due to its nature of publication that suits well with modern process of knowledge appraisal, i.e., 'quick' and 'short'. In this modern digital era, the announcing of new knowledge needs to be 'quick' enough to compete with the fast-pace elimination of old knowledge. This process is made possible by the advance of desktop and mobile internet that allows information to reach all corners of the world within 'clicks'. Any new knowledge or new discovery is almost instantly exposed to debates and reinvestigations upon its release. As a result, even though researchers are constantly racing against time to get their results published and to claim novelty, the novelty is often 'short' in the time span. With the increasing popularity of open-access online journals (Giles, 2007), this process of new knowledge generation and regeneration is becoming a global norm. At the same time, modern readers are also tuned to prefer reading materials that are 'short' enough to be read quickly (Lyytinen et al., 2007). As a result, journal publication is rapidly attracting a bigger pool of modern readers.

The value of journal publication also ties closely with the dominance of bibliometric systems for university and faculty rankings (Ahuillo, Bar-Ilan, Levene, \& Orteja, 2010; Chen \& Liao, 2012; Haustein, Mittermaind, Tunger, \& Julich, 2009). Today, almost all top world university rankings are using bibliometric systems, based predominantly on research publication index and web visibility, as the main objective measures to evaluate university performances and academic influences (Ahuillo et al., 2010; Chen \& Liao, 2012). At various academic levels, journal publication contributes substantially to this numerical game. Therefore, journal publication is now becoming a key indicator used to determine intake, promotion and tenure renewal of academicians at both the faculty and university levels. Productivity in journal publication has been widely investigated (Bentley, 2012; Hemmings \& Kay, 2010; Jonkers \& Tijssen, 2008; Miller, Taylor, \& Bedeian, 2011). So far, researchers had identified several factors such as the quality of doctoral training (Lyytinen et al., 2007), seniority and mentorship (Bentley, 2012), and even gender (Bentley, 2012), as predictors of scholarly outputs. One point worth noting is 
many predictors are contextually bonded (Bentley, 2012; Jonkers \& Tijssen, 2008; Lyytinen et al., 2007), in response to the dynamic and the shaping of institutional, national and regional research and publishing cultures.

Regional difference is especially prominent. Scholars in different regions are surrounded by different research cultures (Jonkers \& Tijssen, 2008; Lyytinen et al., 2007). For example, academicians in North America are typically perceived as experiencing a stronger publishing culture as compared to their other counterparts. The North American academicians are characterized by having better commands of research methods, better writing skills, better understanding of journal genre, and better understanding of the review process (Lyytinen et al., 2007). Comparatively, researchers in some other regions are experiencing lacking in these aspects. Predictors of publication productivity might also vary across the regions. For example, a strong relationship between seniority and publication productivity had been repeatedly found amongst the Australian academicians (Bentley, 2012; Hemmings \& Kay, 2010). However, such relationship was found to be less strong in developing countries such as China where the junior faculty members face higher pressure of securing tenure that uses performance-based measure (Jonkers \& Tijssen, 2008). Such differences highlight the importance to investigate into the local context in the attempt of identifying strategies for improved performance. Therefore, this study was initiated by the dean office of an educational faculty in Malaysia to investigate the local factors that would promote productivity in journal publication amongst the faculty members. The ultimate aim of this study is to identify catalyst strategies to improve the publication index at the faculty level.

\section{METHODOLOGY}

This study was the preliminary investigation conducted by the dean's office prior to the strategic planning. A committee appointed by the dean reviewed the faculty's publication record in Year 2011. The record was traced from the school database contributed by the faculty members as part of their work progress reports. The bibliography list generated from the database was transferred to SPSS version 17.0 for the coding of (1) authors' academic positions, (2) characteristics of authorship and co-authorship, and (3) other factors potentially contributed to successful publication.

\section{RESULTS}

The faculty comprises five professors, 11 associate professors and 52 lecturers (i.e., $\mathrm{N}=68$ ). In 2011, 115 publications were reportedly published by the faculty members. As illustrated in Figure 1, there were 26 publications in ISI and Scopus-listed journals, 64 publications in other journals and 25 other publications (e.g., book, edited book, book chapter, research monograph etc). No correlational relationship was found between publication productivity and seniority.

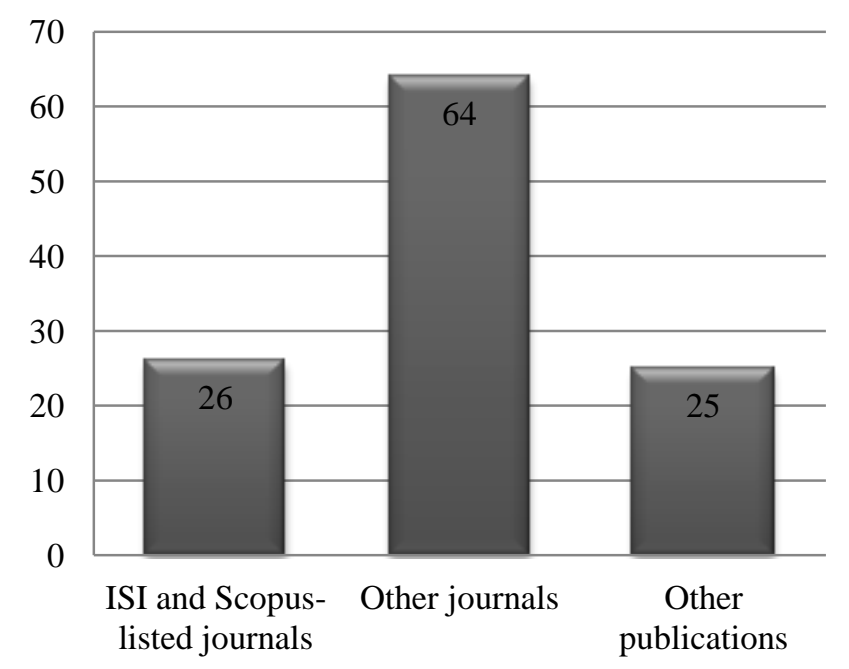

Figure 1 Number of publications in year 2011 
Analyses of the authorship characteristics showed that group authorship was more common than single authorship. For publications in ISI and Scopus-listed journals, there was only one publication produced by a single author $(n=1, N=26,3.8 \%)$. For other journals, there were five publications $(n=5, N=64)$, merely comprises $7.8 \%$ of publications in that group. This set of results suggested that group writing was more popular and potentially it was also more productive amongst the faculty members.

Next, the types of group writings in journal publications were analyzed. The results showed that three quarters of the publications were products of co-writing with research students $(\mathrm{n}=74)$ and the remaining one quarter were products of co-writing with colleagues $(n=24)$. This set of findings pointed to the relevance of research supervision and collegiate collaboration as catalysts of scholarly publications.

\section{CONCLUSION AND RECOMMENDATIONS}

This study aimed to explore the types of publications produced by the members of an education faculty in Malaysia. Through this investigation, the committee from the dean's office hoped to identify factors related to improved performance, and hence to identify the catalysts strategies for pushing up the faculty publication index. The major findings indicated that group writings, i.e., co-writings with research students and colleagues, were important catalysts for successful journal publications amongst the faculty members. These findings helped the committee to identify the directions for future project development.

The first direction would be to provide supports and facilities to encourage co-writings between research supervisors and supervisees. The recommended activities include writing-skills trainings and writing re-treats organized for both lecturers and students. Student research writing awards can be given to those who have published their research works with their research supervisors. As Lyytinen et al. (2007) strongly advocated, the faculty should also develop quality $\mathrm{PhD}$ programs that emphasize good trainings in research methodology, appreciation of theories, and writing skills. At the same time, collegiate collaboration in research grants and research writings can also be promoted at the faculty level via the establishment of research clusters. As part of outcome measures, the faculty can organize an annual expo for different research clusters to showcase their research products and to offer incentives and recognitions to high-performing clusters.

Strategically planned faculty development programs like such had been previously implemented by some American medical faculties and mostly had recorded positive outcomes (Gusic et al., 2010; Sehgal, Sharpe, Auerbach, \& Wachter, 2011). For example, Gusic et al. (2010) outlined nine principles of best practices: (1) provide protected time for project work, (2) ensure projects are aligned with faculty effort assignments, (3) establish a framework for a scholarly approach to projects, (4) provide a formal expectation for scholarship as a project outcome, (5) focus mentoring on participants' needs for their projects, (6) monitor the mentorship relationships/provide support for mentors, (7) provide support during the program to ensure progress, (8) recognize success (formal presentation, certificate, celebration event), and (9) evaluate project outcomes (p.1490). Such principles could be referred and adapted accordingly for the local context. Specific to publications in top-level journals, Lyytinen et al. (2007) urge faculties to invest in research culture that values reviewing amongst the faculty members and reviewing for top-level journals. They believe that reviewing and editing in journals are important exercises for improved scholarship in writing. These ideas are rather universal and potentially transferable across settings. Therefore, we also seek to integrate these ideas as needed in the future project development.

\section{AUTHOR INFORMATION}

Low Hui Min, Ph.D., School of Educational Studies, Universiti Sains Malaysia, 11800 Minden Pulau Pinang, Malaysia. E-mail: lowhm@usm.my (Corresponding author)

Amelia Abdullah, Ph.D., School of Educational Studies, Universiti Sains Malaysia, 11800 Minden Pulau Pinang, Malaysia. E-mail: amelia@usm.my

Abdul Rashid Mohamed, Ph.D., Dean, School of Educational Studies, Universiti Sains Malaysia, 11800 Minden Pulau Pinang, Malaysia. E-mail: rich@usm.my 


\section{REFERENCES}

1. Ahuillo, I. F., Bar-Ilan, J., Levene, M., \& Orteja, J. L. (2010). Comparing university rankings. Scientometrics, 85, 243-256.

2. Bentley, P. (2012). Gender differences and factors affecting publication productivity among Australian university academics. Journal of Sociology, 48(1), 85-103.

3. Chen, K., \& Liao, P. (2012). A comparative study on world university rankings: A bibliometric survey. Scientometrics, 92, 89-103.

4. $\quad$ Giles, J. (2007). Open-access journal will publish first, judge later. Nature, 445(4), 9.

5. Gusic, M. E., Milner, R. J., Tisdell, E. J., Taylor, E. W., Quillen, D. A., \& Thorndyke, L. E. (2010). The essential value of projects in faculty development. Academic Medicine, 85(9), 1484-1490.

6. Haustein, S., Mittermaind, B., Tunger, D., \& Julich, F. (2009). Bibliometrische Analyse AsiatischPazifischer Raum [Asia Pacific bibliometric analysis]: International Bureau of BMBF.

7. Hemmings, B., \& Kay, R. (2010). Journal ratings and the publications of Australian academics. Issues in Educational Research, 20(234-243).

8. Jonkers, K., \& Tijssen, R. (2008). Chinese researchers returning home: Impact of international mobility on research collaboration and scientific productivity. Scientometrics, 77(2), 309-333.

9. Lyytinen, K., Baskerville, R., Livari, J., \& Te'eni, D. (2007). Why the old world cannot publish? Overcoming challenges in publishing high-impact IS research. European Journal of Information Systems, 16, 317-326.

10. Miller, A. N., Taylor, S. G., \& Bedeian, A. G. (2011). Publish or perish: Academic life as management faculty live it. Career Development International, 16(5), 422-445.

11. Sehgal, N. L., Sharpe, B. A., Auerbach, A. A., \& Wachter, R. M. (2011). Investing in the future: Building an academic hospitalist faculty development program. Journal of Hospital Medicine, 6(3), 161-166. 